\title{
A pinching problem for locally homogeneous spaces
}

\author{
By Atsushi KATsudA
}

(Received May 13, 1985)

(Revised June 13, 1987)

It is well known that a Riemannian manifold is locally symmetric if and only if the curvature tensor is parallel. In 1958, W. Ambrose and I. M. Singer [1] gave a local characterization of locally homogeneous spaces, which is an extension of locally symmetric case.

In this note, we consider the following problem,

If the local property of a Riemannian manifold is similar to the above space, then is it diffeomorphic to the above?

Initiated by $\mathrm{H}$. Rauch, many works for such a kind of problem are developed. Recently, M. Gromov gave a new aspect and showed a remarkable theorem. We use this theorem as a main tool. The same method is used in [2], [6], [8] etc.

For an $n$-dimensional Riemannian manifold $(M, g)$, we denote by $\nabla$ the covariant derivative, $R=R_{M}$ the curvature tensor, $K_{M}$ the sectional curvature, $\operatorname{vol}(M)$ the volume, $\operatorname{diam}(M)$ the diameter of $M$. Let $\mathfrak{M}(n, \Lambda, V, D)$ be the category of all complete Riemannian manifolds $M$ with dimension $n,\left|K_{M}\right| \leqq \Lambda^{2}$, $\operatorname{vol}(M) \geqq V, \operatorname{diam}(M) \leqq D$. We shall prove the following Theorems 1 and 2 .

TheOREM 1. Given integers $m, n>0$ and constants $\Lambda, V, D>0$, there is a constant $\delta=\delta(m, n, \Lambda, V, D)>0$ depending only on $m, n, \Lambda, V, D$ such that if $M \in$ $\mathfrak{M}(n, \Lambda, V, D)$ satisfies the condition that $\left|\nabla^{m} R\right| \leqq \delta$, then $M$ is diffeomorphic to $a$ locally symmetric space.

It should be noted that Min-Oo and E. Ruh [13], [14] gave other conditions of pinching for locally symmetric space. In their assumption, the constant does not depend on the volume or the injectivity radius. In our case, the almost flat manifold is an example to show that removing the dependence of the volume is impossible. This fact is noted by Ruh.

THEOREM 2. Given an integer $n>0$ and constants $\Lambda, V, D>0$, there is a constant $\delta=\delta(n, \Lambda, V, D)>0$ depending only on $n, \Lambda, V, D$ such that if $M \in M(n, \Lambda, V, D)$ has a tensor field $T$ of type $(1,2)$ satisfying the following conditions (*), then $M$ is diffeomorphic to a locally homogeneous space. 
(1) $g\left(T_{X} Y, T_{X} Y\right) \leqq \Lambda^{2}$

(2) $\left|g\left(T_{X} Y, Z\right)+g\left(Y, T_{X} Z\right)\right| \leqq \delta$

$(*)$

(3) $\left|\left(\nabla_{X} R\right)_{Y Z}-\left[T_{X} Y, R_{Y Z}\right]+R_{T_{X} Y Z}+R_{Y T_{X} Z}\right| \leqq \delta$

(4) $\left|\left(\nabla_{X} T\right)_{Y}-\left[T_{X}, T_{Y}\right]+T_{T_{X}}\right| \leqq \delta$

for unit vectors $X, Y, Z$.

W. Ambrose and I. M. Singer [1] proved that if $M$ satisfies (*) for $\delta=0$, then $M$ is isometric to a locally homogeneous space. In this case, $T$ is a difference between the Levi-Civita connection and the canonical connection of $M$. If $T=0$, then $M$ is locally symmetric.

Acknowledgement. The author is indebted to Professors A. Morimoto and T. Sunada. He also wishes to thank the referee for useful advices.

\section{$\S 1$. Preliminaries.}

We summarize basic tools for the proof of theorems. Let $\mathfrak{M}(n, \Lambda, i)$ be the category of all complete Riemannian manifolds with dimension $n,\left|K_{M}\right| \leqq \Lambda^{2}$ and the injectivity radius $i_{M} \geqq i$. For $M, M^{\prime} \in \mathfrak{M}(n, \Lambda, i)$, we recall the definition of Lipschitz and Hausdorff distances.

Definition ([9]). 1. Lipschitz distance; $d_{L}\left(M, M^{\prime}\right)$.

$$
d_{L}\left(M, M^{\prime}\right)=\inf \left(|\log (d f)|+\left|\log \left(d f^{-1}\right)\right|\right),
$$

where infimum runs over bi-Lipschitz homeomorphisms $f: M \rightarrow M^{\prime}$ and

$$
d f=\sup \{d(f(x), f(y)) / d(x, y) \mid x, y \in M, x \neq y\}
$$

and $d(x, y)$ is the distance induced from the Riemannian metric.

2. Hausdorff distance; $d_{H}\left(M, M^{\prime}\right)$.

$$
d_{H}\left(M, M^{\prime}\right)=\inf \left(d_{H}^{Z}\left(f(M), f^{\prime}\left(M^{\prime}\right)\right)\right),
$$

where infimun runs over metric spaces $Z$ with a distance $d^{Z}$, isometric imbeddings $f: M \rightarrow Z, f^{\prime}: M^{\prime} \rightarrow Z$ and

$$
d_{H}^{Z}\left(f(M), f^{\prime}\left(M^{\prime}\right)\right)=\max \left(\sup _{x \in f(M)}\left(\inf _{y \in f^{\prime}\left(M^{\prime}\right)} d(x, y)\right), \sup _{y \in f^{\prime}\left(M^{\prime}\right)}\left(\inf _{x \in f(M)} d(x, y)\right)\right) .
$$

The following theorems are essential for the proof.

THEOREM A ([9]). $\mathfrak{M}(n, \Lambda, V, D)$ is precompact with respect to the Hausdorff distance. 
Theorem B ([7], [9], [11], [18]). If $\left\{M_{i}\right\} \subset \mathfrak{M}(n, \Lambda, i)$ is a Cauchy sequence with respect to the Hausdorff distance, then there is a smooth manifold $M$ with $C^{1, \alpha}$-metric such that

(i) $M_{i}$ is diffeomorphic to $M$ for sufficiently large $i$,

(ii) $\lim d_{L}\left(M_{i}, M\right)=\lim d_{H}\left(M_{i}, M\right)=0$.

THEOREM C. If $\left\{M_{i}\right\} \subset \mathfrak{M}(n, \Lambda, i)$ is a Cauchy sequence with respect to the Hausdorff distance and there is a constant $\Lambda^{\prime}>0$ with $\left|\nabla R_{M_{i}}\right| \leqq \Lambda^{\prime}$, then there exists a subsequence of $\left\{M_{i}\right\}$ such that the exponential map of $M$ is of class $C^{1}$.

Theorem $C$ is necessary for the proof of the following theorem. The proof is essentially contained in [7].

THEOREM D ([12], I, Theorem 3.10). If the exponential map of a Riemannian manifold $M$ is of class $C^{1}$, a distance preserving selfmap of $M$ is of class $C^{1}$.

THEOREM E ([15], p. 200). Let $G$ be a locally compact effective transformation group of connected $C^{1}$ manifold $M$ and let each transformation of $G$ be of class $C^{1}$. Then $G$ is a Lie group and the map $G \times M \rightarrow M$ is of class $C^{1}$.

\section{$\S 2$. Outline of the proof.}

Assume that the conclusion of Theorem 2 does not hold. Then, there exists a sequence $\left\{M_{i}\right\} \subset \mathfrak{M}(n, \Lambda, V, D)$ such that

(1) $M_{i}$ satisfies the condition (*) in Theorem 2 for $\delta=1 / i$,

(2) $M_{i}$ is not diffeomorphic to a locally homogeneous space.

From Theorem A, taking a subsequence if necessary, we may assume that $\left\{M_{i}\right\}$ is a Cauchy sequence with respect to the Hausdorff distance. Then, by Theorems $\mathrm{B}$ and $\mathrm{C}$, there is a limit manifold $M$ with the exponential map of class $C^{1}$. Assume for a moment the following condition ( $F$ ) is proved.

(F) The identity component of the group of isometries $G_{0}$ of the universal covering space $\tilde{M}$ of $M$ acts transitively on $\tilde{M}$.

Then, by Theorems D and E, $G_{0}$ is a Lie group and $\tilde{M}$ is $C^{1}$-diffeomorphic to the homogeneous space $G_{0} / K$, where $K$ is the isotropy subgroup of $G_{0}$. Since $\tilde{M}$ is $C^{\infty}$-manifold and $G_{0} / K$ is so, these are $C^{\infty}$-diffeomorphic. The induced metric $g_{0}$ of $G_{0} / K$ from $C^{1, \alpha}$-metric is $G_{0}$-invariant, thus $C^{\infty}$. Using Theorem $\mathrm{B}$, we see $M_{i}$ is diffeomorphic to a locally homogeneous space. This is a contradiction.

To prove $(\mathrm{F})$, we first see that there is a local quasi-isometry from a point $p$ to $q$ in $M$ by the argument in [12]. Next, extend this local quasi-isometry to of larger domain by the method of Cartan-Ambrose-Hicks. Finally, Ascoli's 
theorem implies the condition $(\mathrm{F})$.

Note that the condition $\left|K_{M}\right| \leqq \Lambda^{2}$ and $\left|\nabla^{m} R_{M}\right| \leqq \delta^{\prime}$ for $m \geqq 2$ implies $|\nabla R|$ $\leqq \delta$ by interpolation inequality. Thus, the proof of Theorem 1 is the special case of Theorem 2 with $T=0$.

For the sake of the brevity, fix $\mathfrak{M}(n, \Lambda, v, D)$ or $\mathfrak{M}(n, \Lambda, i)$ and a manifold $M$ always belongs to this category. (Note that by Cheeger's result, we may assume $\mathfrak{M}(n, \Lambda, v, D) \subset \mathfrak{M}(n, \Lambda, i)$ for some $i>0$.) The dependence of $n, \Lambda, v, D, i$ in the constants which appear in later abbreviated. Moreover, in the following sections, we use the same letter $\delta$ or $C$ in different constants, unless otherwise stated, $\delta$ (resp. $C$ ) appearing in later is smaller (resp. larger) than the previous one.

\section{§3. Comparison lemmas between the Levi-Civita connection and the canonical connection.}

In later sections, we use two kind of connections. One is the Levi-Civita connection $\nabla$ and the other is the connection $\tilde{\nabla}=\nabla-T$, which is the canonical connection if $M$ is locally homogeneous.

Put the curvature $\tilde{R}$ of $\tilde{\nabla}, \tilde{R}_{X Y}=\tilde{\nabla}_{[X, Y]}-\left[\tilde{\nabla}_{X}, \tilde{\nabla}_{Y}\right]$ and the torsion $S$ of $\tilde{\nabla}$, $S_{X} Y=T_{X} Y-T_{Y} X$. Then, the condition $(*)$ for $\delta$ in Theorem 2 is equivalent to the following condition $(* *)$ for some $\delta^{\prime}$. Note that $\delta^{\prime}$ tends to zero when $\delta$ tends to zero.

(1) $|T| \leqq \Lambda$

(2) $|\tilde{\nabla} g| \leqq \delta^{\prime}$

(3) $|\tilde{\nabla} \tilde{R}| \leqq \delta^{\prime}$

(4) $\quad|\tilde{\nabla} T| \leqq \delta^{\prime} \quad$ (i. e. $\left.|\tilde{\nabla} S| \leqq \delta^{\prime}\right)$

where $|\cdot|$ is the norm induced from Riemannian metric $g$. (In later, we use same letter $\delta$ in the conditions $(*)$ and $(* *)$ for simplicity.)

Let $l(\gamma)$ denote the length of a curve $\gamma$. We call a geodesic, the exponential map with respect to the connection $\tilde{\nabla}$ (resp. $\nabla$ ), $\tilde{\nabla}$-geodesic (resp. $\nabla$-geodesic), $\tilde{\nabla}$-exp or $\exp (\tilde{\nabla})($ resp. $\nabla$-exp or $\exp (\nabla))$ etc. For any points $p, q \in M$, let $\tilde{P}_{p q}^{\gamma}$ (resp. $P_{p q}^{r}$ ) be the parallel translation with respect to the connection $\tilde{\nabla}$ (resp. $\nabla$ ) along a curve $\gamma$ from $p$ to $q$. In particular, if $\gamma$ is minimal $\nabla$-geodesic then we denote $\tilde{P}_{p q}=\tilde{P}_{p q}^{r}$ (resp. $P_{p q}=P_{p q}^{r}$ ).

First we give an estimate of the norm of the $\tilde{\nabla}$-parallel transformation.

LEMmA 3.1. For any $\varepsilon>0$ and $R>0$, there exists a constant $\delta=\delta(\varepsilon, R)>0$ such that if $M$ satisfies the condition (*) or (**) for $\delta$ and a curve $\gamma$ in $M$ from $p$ to $q$ satisfies $l(\gamma) \leqq R$, then 


$$
|| P_{p q}^{r} X|-1| \leqq \varepsilon
$$

for a unit vector $X$ in $T_{p} M$.

PRoof. Parametrize $\gamma$ by arclength and put $X_{t}=\tilde{P}_{p \gamma(t)}^{\gamma} X$. Then,

$$
\begin{aligned}
\left|\frac{d}{d t} g\left(X_{t}, X_{t}\right)\right|=2\left|g\left(\nabla_{\dot{\gamma}(t)} X_{t}, X_{t}\right)\right| & \leqq 2\left(\left|g\left(\tilde{\nabla}_{\dot{\gamma}(t)} X_{t}, X_{t}\right)\right|+\left|g\left(T_{\dot{\gamma}(t)} X_{t}, X_{t}\right)\right|\right) \\
& \leqq 2\left|g\left(T_{\dot{\gamma}(t)} X_{t}, X_{t}\right)\right| \leqq 2 \delta g\left(X_{t}, X_{t}\right) .
\end{aligned}
$$

This implies

Hence the conclusion.

$$
e^{-2 \delta t} \leqq\left|g\left(X_{t}, X_{t}\right)\right| \leqq e^{2 \delta t}
$$

Second we give an estimate of the length of $\tilde{\nabla}$-geodesics.

LEMmA 3.2. For any $\varepsilon>0$ and $R>0$, there exists a constant $\delta(\varepsilon, R)>0$ such that if $M$ satisfies the condition (*) or (**) for $\delta$ and $a \tilde{\nabla}$-geodesic $\gamma$ in $M$ with $\gamma(0)$ $=p,|\dot{\gamma}(0)|=1$, then

$$
|| \dot{\gamma}(t)|-1| \leqq \varepsilon \quad \text { for } 0 \leqq t \leqq R .
$$

Proof. Put $f(t)=|\dot{\gamma}(t)|^{2}$. Then,

$$
\frac{d}{d t} f(t)=\left|2 g\left(\nabla_{\dot{\gamma}(t)} \dot{\gamma}(t), \dot{\gamma}(t)\right)\right|=\left|2 g\left(T_{\dot{\gamma}(t)} \dot{\gamma}(t), \dot{\gamma}(t)\right)\right| \leqq 2 \delta f^{3 / 2}(t) .
$$

This implies the conclusion.

Third, we estimate the injectivity radius $r=r_{M}$ of $M$ with respect to the connection $\tilde{\nabla}$. Note that the connection $\tilde{\nabla}$ is not always complete $([\mathbf{1 2}], \mathrm{I}, \mathrm{p}$. 139). The $\tilde{\nabla}$-exp can only be defined in the neighborhood of the origin in general.

Proposition 3.3. Assume that $M$ satisfies the condition (*) or (**) for $\delta$. There exists a constant $0<r=r(\boldsymbol{\delta})\left(<i_{M}\right)$ such that $\exp _{p}(\tilde{\nabla})$ is injectively mapped onto $B_{r}(p)$.

PROOF. Recall the Jacobi equation with respect to $\tilde{\nabla}$,

$$
\tilde{\nabla}_{\dot{c}}^{2} J+\tilde{\nabla}_{\dot{c}} S(J, \dot{c})+\widetilde{R}(J, \dot{c}) \dot{c}=0
$$

where $c$ is $\tilde{\nabla}$-geodesic. Combining with Lemma 3.2, there exists $r_{1}>0$ such that if $X \in T B_{r_{1}}(p)$, then

$$
1 / 2 \leqq\left\|d_{X} \exp _{p}\right\| \leqq 2
$$

and

$$
\exp _{p}(\tilde{\nabla})\left(T B_{r_{1}}(p)\right) \subset B_{i_{M}}(p) \equiv B
$$


We show that $\exp _{p}(\tilde{\nabla}) \equiv \exp$ is injective on $T B_{r_{1}}(p)$. Assume that there exist $X_{1}, X_{2} \in T B_{r_{1}}(p)$ such that $\exp X_{1}=\exp X_{2}=q$. Put $c_{i}(t)=\exp t X_{i}$. Since $B$ is simply connected, there exists a homotopy $F(s, t):[0,1] \times\left[0, l_{s}\right] \rightarrow B$ with $F_{0}(t)$ $=c_{1}(t), F_{1}(t)=c_{2}(t)$. Since the image of $F$ is compact, there exists $G(s, t):[0,1]$ $\times\left[0, l_{s}\right] \rightarrow T_{p} M$ such that $\exp (G(s, t))=F(s, t)$. But $\exp \left(G\left(s, l_{s}\right)\right)=q$, this contradicts (3.3.1).

Next, we find that there exists $r>0$ such that $B_{r}(p) \subset \exp \left(T B_{r_{1}}(p)\right)$. Take a minimal $\nabla$-geodesic $c_{1}(t)$ in $T B_{r_{1}}(p)$ with $c_{1}(0)=p, c_{1}(l)=q \in \partial\left(\exp \left(T B_{r_{1}}(p)\right)\right.$. Let $c_{2}(t)=\exp ^{-1}\left(c_{1}(t)\right)$. Then, by Lemma 3.2 and (3.3.1), we see

$$
r_{1} \leqq l\left(c_{2}\right) \leqq 2 l \text {. }
$$

Therefore we get the conclusion by taking $r<r_{1} / 2$.

Finally, we estimate the difference of $\tilde{\nabla}$-parallel translations and $\nabla$-parallel translations. For a curve $\gamma$ in $M$ with $\gamma(0)=p,|\dot{\gamma}(t)|=1, l(\gamma) \leqq R$ and a unit vector $X$ in $T_{p} M$, let $X_{t}=\tilde{P}_{p_{\gamma}(t)}^{\gamma} X, Y_{t}=P_{p \gamma(t)}^{r} X$.

LEMMA 3.4. $\left|g\left(X_{t}, Y_{t}\right)\right| \leqq 2 \Lambda t \quad$ for $0 \leqq t \leqq R$.

Proof. By Lemma 3.2, we have

$$
\begin{aligned}
\left|\frac{d}{d t} g\left(X_{t}, Y_{t}\right)\right|=\left|g\left(\nabla_{\dot{\gamma}(t)} X_{t}, Y_{t}\right)\right| & \leqq\left|g\left(\tilde{\nabla}_{\dot{\gamma}(t)} X_{t}, Y_{t}\right)\right|+\left|g\left(T_{\dot{\gamma}(t)} X_{t}, Y_{t}\right)\right| \\
& \leqq\left|g\left(T_{\dot{\gamma}(t)} X_{t}, Y_{t}\right)\right| \leqq \Lambda(1+\delta) \leqq 2 \Lambda .
\end{aligned}
$$

This implies

$$
\left|g\left(X_{t}, Y_{t}\right)\right| \leqq 2 \Lambda t
$$

q.e.d.

\section{$\S 4$. Construction of local quasi-isometry.}

For $p, q \in M$, we give a local quasi-isometry $\phi$ from a neighborhood $U$ of $p$ to a neighborhood $V$ of $q$. In the case when $M$ satisfies the condition (*) for $\delta=0$, the corresponding result is proved in [12], I, p. 256-262. Take a continuous curve $\sigma:[0, l] \rightarrow M$ such that $\sigma(0)=p, \sigma(l)=q, l(\sigma)=l \leqq R$ and set $I=\tilde{P}_{p q}^{\sigma}$. We define a map $\phi: U=B_{r / 2}(p) \rightarrow V$ by $\phi=\exp _{q}(\tilde{\nabla}) \circ I \circ \exp _{p}(\tilde{\nabla})^{-1}$, where $r=r_{M}$.

Proposition 4.1. Given $\varepsilon>0$ and $R>0$, there exists $\delta=\delta(R, \varepsilon)>0$ such that if $M$ satisfies the conditions (*) or (**) for $\delta$, then the map $\phi$ satisfies the following;

$$
\begin{aligned}
& \left|\tilde{P}_{\phi(x) q^{\circ}}^{\phi(\gamma)} d \phi_{x}(X)-d \phi_{p} \circ \tilde{P}_{x p}^{\gamma}(X)\right| \leqq \varepsilon \\
& || d \phi_{x}(X)|-1| \leqq \varepsilon
\end{aligned}
$$

for each unit vector $X$ of $T_{p} M$, where $\gamma$ is a $\nabla$-geodesic from $x$ to $p$ in $U$.

Proof. Take $\tilde{\nabla}$-normal coordinates $\left\{x_{i}\right\}$ at $p$ and $\left\{y_{i}\right\}$ at $q$ such that 
$I\left(\left(\partial / \partial x_{i}\right)_{p}\right)=\left(\partial / \partial y_{i}\right)_{q}, g\left(\left(\partial / \partial x_{i}\right)_{p},\left(\partial / \partial x_{j}\right)_{p}\right)=\delta_{i j} . \quad$ Note that $x^{i}=y^{i} \circ \phi$. Let $L(M)$ be a linear frame bundle over $M$. We call a section $\sigma: U \rightarrow L(M)$ is adapted to the normal coordinates $\left\{x_{i}\right\}$ when $\sigma(x)$ is the frame obtained by the $\tilde{\nabla}$-parallel translation of the linear frame at the origin along the radial $\tilde{\nabla}$-geodesic. Let $\theta=\left(\theta^{i}\right), \omega=\left(\omega^{i}\right), \Theta=\left(\Theta^{i}\right)$ and $\Omega=\left(\Omega^{i}{ }_{j}\right)$ be the canonical form, the connection form, the torsion form and the curvature form of $L(M)$ respectively. Put

$$
\begin{aligned}
& \bar{\theta}^{i}=\sigma^{*} \theta=\sum A^{i}{ }_{j} d x^{j}, \\
& \overline{\omega^{i}{ }_{j}}=\sigma^{*} \omega^{i}{ }_{j}=\sum B^{i}{ }_{j k} d x^{k}, \\
& \overline{\Theta^{i}}=\sigma^{*} \Theta^{i}=\sum \frac{1}{2} S_{j k}^{i} \overline{\theta^{i}} \wedge \overline{\theta^{j}}, \\
& \overline{\Omega^{i}{ }_{j}}=\sigma^{*}{\Omega^{i}}_{j}=\sum \frac{1}{2} R^{i}{ }_{j k} \overline{\theta^{k}} \wedge \overline{\theta^{l}} .
\end{aligned}
$$

Fix $a=\left(a^{1}, \cdots, a^{n}\right)$, we put

$$
\begin{array}{ll}
\hat{A}^{i}{ }_{j}(t)=t A^{i}{ }_{j}(t a), & \hat{B}^{i}{ }_{j k}(t)=t B^{i}{ }_{j k}(t a), \\
\hat{S}^{i}{ }_{j k}=S^{i}{ }_{j k}(t a), & \hat{R}^{i}{ }_{j k l}(t)=R^{i}{ }_{j k l}(t a) .
\end{array}
$$

Then from [12], I, p. 258, Lemma 2, these satisfy the following system of ordinary differential equations,

$$
\begin{aligned}
& d \hat{A}^{i}{ }_{j} / d t=\delta^{i}{ }_{j}+\sum \hat{B}^{i}{ }_{l j} a^{l}+\sum \hat{S}^{i}{ }_{l m} \hat{A}^{m}{ }_{j} a^{l}, \\
& d \hat{B}^{i}{ }_{j k} / d t=\sum \hat{R}^{i}{ }_{j l m} \hat{A}^{m}{ }_{k} a^{l}, \\
& \hat{A}^{i}{ }_{j}(0)=0, \quad \hat{B}^{i}{ }_{j k}(0)=0 .
\end{aligned}
$$

Let $u(t)$ be a horizontal lift of a radial $\tilde{\nabla}$-geodesic $c(t)=\left(t a^{i}\right)$ to $L(M)$ with $u(0)=\left(\left(\partial / \partial x^{1}\right)_{p}, \cdots,\left(\partial / \partial x^{n}\right)_{p}\right)$. Then, from [12], I, p. 260, Lemma 1, we have

$$
\sum\left(d \hat{S}^{i}{ }_{j k} / d t\right)^{2}=\left|u^{-1}(t)\left(\tilde{\nabla}_{i(t)} S\right)\right|^{2}
$$

where $u(t)$ is considered as a linear mapping from $\boldsymbol{R}^{n}$ to $T_{u(t)} M$. Since the operator norm of $u(t)$ can be estimated by Lemma 3.1, we have that if $t$ is smaller than $r$ and $\delta$ in Lemma 3.2, then,

$$
\left|u^{-1}(t)\left(\tilde{\nabla}_{\dot{c}(t)} S\right)\right| \leqq(1+\varepsilon r)|\tilde{\nabla} S||\dot{c}(t)| \leqq(1+\varepsilon r)(1+\varepsilon) \delta .
$$

Therefore, taking $\varepsilon<\min (1 / 2 r, 1 / 2)$, we get

$$
\left|\hat{S}^{i}{ }_{j k}(t)-\hat{S}_{j k}{ }_{j k}(0)\right| \leqq 2 \delta t \text {. }
$$

Similarly, we also have

$$
\left|\hat{R}_{j k l}^{i}(t)-\hat{R}_{j k l}^{i}(0)\right| \leqq 2 \delta t .
$$

Replace $x_{i}$ by $y_{i}$ in the definition of $\hat{A}^{i}{ }_{j}, \hat{B}^{i}{ }_{j k}, \hat{S}^{i}{ }_{j k}, \hat{R}^{i}{ }_{j k l}$ and let $\hat{A}^{\prime i}{ }_{j}, \hat{B}^{\prime i}{ }_{j k}$, 
$\hat{S}^{\prime i}{ }_{j k}, \hat{R}^{\prime i}{ }_{j k l}$ denote the resulting functions respectively. Note that

and

$$
\begin{aligned}
& \hat{S}^{i}{ }_{j k}(0)=\left\langle S\left(\partial / \partial x^{j}, \partial / \partial x^{k}\right), \partial / \partial x^{i}\right\rangle, \\
& \hat{S}^{\prime}{ }_{j k}(0)=\left\langle S\left(I\left(\partial / \partial x^{j}\right), I\left(\partial / \partial x^{k}\right)\right), I\left(\partial / \partial x^{i}\right)\right\rangle,
\end{aligned}
$$

$$
\begin{aligned}
& \hat{R}^{i}{ }_{j k l}(0)=\left\langle R\left(\partial / \partial x^{j}, \partial / \partial x^{k}\right) \partial / \partial x^{l}, \partial / \partial x^{i}\right\rangle, \\
& \hat{R}^{\prime i}{ }_{j k l}(0)=\left\langle R\left(I\left(\partial / \partial x^{j}\right), I\left(\partial / \partial x^{k}\right)\right) I\left(\partial / \partial x^{l}\right), I\left(\partial / \partial x^{i}\right)\right\rangle
\end{aligned}
$$

By an argument similar to the proof of (4.1.4) and (4.1.5), we have

$$
\left|\hat{S}^{\prime i}{ }_{j k}(0)-\hat{S}^{i}{ }_{j k}(0)\right| \leqq 2 \delta R, \quad\left|\hat{R}^{\prime i}{ }_{j k l}(0)-\hat{R}^{i}{ }_{j k l}(0)\right| \leqq 2 \delta R .
$$

Combining with (4.1.4), (4.1.5), we get

$$
\left|\hat{S}^{\prime i}{ }_{j k}(t)-\hat{S}^{i}{ }_{j k}(t)\right| \leqq 4 \delta R, \quad\left|\hat{R}^{\prime i}{ }_{j k l}(t)-\hat{R}^{i}{ }_{j k l}(t)\right| \leqq 4 \delta R .
$$

Since $\hat{A}^{i}{ }_{j}, \hat{A}^{\prime}{ }_{j}, \hat{B}^{i}{ }_{j k}$ and $\hat{B}^{\prime i}{ }_{j k}$ are solutions of (4.1.2), this implies

$$
\left|\hat{A}^{\prime i}{ }_{j}(t)-\hat{A}^{i}{ }_{j}(t)\right| \leqq C \delta R, \quad\left|\hat{B}^{\prime i}{ }_{j k}(t)-\hat{B}^{i}{ }_{j k}(t)\right| \leqq C \delta R
$$

where a constant $C$ depends only on $\delta$ and $R$. From (4.1.1), we have

$$
\left|\overline{\theta^{i}}-\phi^{*} \overline{\theta^{i}}\right| \leqq C \delta R, \quad\left|\overline{\omega_{j}^{i}}-\phi^{*} \overline{\omega^{i}}{ }_{j}\right| \leqq C \delta R .
$$

By [12], I, p. 261, we see

$$
\sigma^{*} \theta(X)=\theta(\sigma X)=\sigma(x)^{-1} X \quad \text { for } \quad X \in T_{x} M .
$$

Therefore, from (4.1.7), we have

$$
\left|\sigma(x)^{-1} X-\sigma(\phi(x))^{-1} d \phi_{x}(X)\right| \leqq C \delta R|X| .
$$

This implies

and

$$
\left|\omega_{j}^{i}-\phi^{*} \omega^{i}{ }_{j}\right| \leqq C \delta R
$$

$$
\left|d \phi\left(\tilde{\nabla}_{x} Y\right)-\tilde{\nabla}_{d \phi_{x}(X)} d \phi_{x}(X)\right| \leqq C \delta R(\max (|X|,|Y|)) .
$$

Now we prove (i). Let $Y_{t}$ be a $\tilde{\nabla}$-parallel vector field along $\gamma$. We put

$$
P_{t}=\tilde{P}_{\phi(\gamma(t)) q}^{\phi(\gamma-1)}, \quad G(t)=P_{t} d \phi_{\gamma(t)}\left(Y_{t}\right)-d \phi_{p}\left(\tilde{P}_{\gamma(t) p}^{r} Y_{t}\right), \quad g(t)=|G(t)|^{2}
$$

where $\gamma^{-1}(t)=\gamma(l-t)$. Then, from Lemma 3.1 and (4.1.8), we get

$$
\begin{aligned}
\left|\frac{d}{d t} g(t)\right| & =\left|2 g\left(\frac{d}{d t}\left(P_{t} d \phi_{\gamma(t)}\left(Y_{t}\right)\right), G(t)\right)\right| \\
& \leqq 2 \sqrt{g(t)}\left|P_{t} \tilde{\nabla}_{d \phi_{\gamma(t)}(\dot{\gamma}(t))} d \phi_{\gamma(t)}\left(Y_{t}\right)\right| \\
& \leqq 2 \sqrt{g(t)}\left(\left|P_{t} d \phi_{\gamma(t)}\left(\tilde{\nabla}_{\dot{\gamma}(t)} Y_{t}\right)\right|+C\left\|P_{t}\right\| \delta R\right) \\
& \leqq 2 C \sqrt{g(t)}\left\|P_{t}\right\| \delta R \leqq C \delta R \sqrt{g(t)}
\end{aligned}
$$


where $\|\cdot\|$ is the norm of the linear operator, and

This implies,

$$
g(0)=0 \text {. }
$$

$$
g(t) \leqq(C \delta R)^{2}
$$

and shows (i) by taking $\delta \leqq \varepsilon / C R$. To prove (ii), we see

$$
\begin{aligned}
|| d \phi_{x}(X)|-1| \leqq & || P_{t} d \phi_{\gamma(t)}\left(Y_{t}\right)|-| d \phi_{x}(X)|| \\
& +|| P_{t} d \phi_{\gamma^{(}(t)}\left(Y_{t}\right)|-| I^{\circ} \tilde{P}_{x p}^{\tau}(X)||+|| I \circ \tilde{P}_{x p}^{\tau}|-1| \\
\leqq & C \delta R \quad \text { for }|X|=1 .
\end{aligned}
$$

Then taking $\delta \leqq \varepsilon / C R$, we get the conclusion.

\section{$\S 5$. The extension of quasi-isometry.}

In this section we prepare several lemmas for an extension of quasi-isometry in Proposition 4.1. The method is similar to the proof of Cartan-Ambrose-Hicks theorem. For $p, q \in M$ with $d(p, q) \leqq R$ and $x, y \in B_{r / 2}(p)$, we define $\nabla$-geodesics $\gamma_{1}, \gamma_{2}, \gamma_{3}$ parametrized by arclength such that $\gamma_{1}(0)=\gamma_{2}(0)=p, \gamma_{1}\left(l_{1}\right)=y, \gamma_{2}\left(l_{2}\right)=x$, $\gamma_{3}(0)=x, \gamma_{3}\left(l_{3}\right)=y$. Put

$$
\begin{aligned}
& \varphi=\exp _{q}(\tilde{\nabla}) \circ I \circ \exp _{p}(\tilde{\nabla})^{-1}, \\
& \phi=\exp _{\varphi(x)}(\tilde{\nabla}) \circ \tilde{P}_{q \varphi\left(\gamma_{2}\right)}^{\varphi\left(\gamma_{2}\right)} \circ I^{\circ} \tilde{P}_{x p}^{\gamma_{1}-1} \circ \exp _{p}(\tilde{\nabla})^{-1},
\end{aligned}
$$

where $I=\tilde{P}_{p q}^{\sigma}$ in Section 4 . Let $\bar{\gamma}_{3}, \tilde{\gamma}_{3}$ be $\nabla$-geodesics with $\bar{\gamma}_{3}(0)=\varphi(x), \bar{\gamma}_{3}\left(\bar{l}_{3}\right)=\varphi(y)$ and $\tilde{\gamma}_{3}(0)=\varphi(x), \dot{\tilde{\gamma}}_{3}(0)=\tilde{P}_{q \varphi(x)}^{\varphi\left(\gamma_{2}\right)} \circ I \circ \tilde{P}_{x p}\left(\dot{\gamma}_{3}(0)\right)$. Then,

LEMMA 5.1. For any $\varepsilon>0$, there exists $\delta=\delta(\varepsilon)>0$ such that if $M$ satisfies $(*)$ or $(* *)$ for $\delta$, then,

$$
\left|g\left(\dot{\bar{\gamma}}_{3}(0), \dot{\tilde{\gamma}}_{3}(0)\right)-1\right| \leqq \varepsilon .
$$

Proof. Take a $C^{1}$-curve $\tau$ in $M$ such that $\tau(0)=\varphi(y), \dot{\tau}(0)=-d \varphi\left(\dot{\gamma}_{3}\left(l_{3}\right)\right)$, $\tau(l)=\varphi(x),\left.\tau\right|_{[-1 / 1,0]}$ is $\nabla$-geodesic parametrized by proportional to arclength, (not necessary unit speed) and $\tau(t)=\varphi\left(\gamma_{3}\left(l_{3}-t\right)\right)$ for $t>0$. By Proposition 4.1,

$$
|| \dot{\tau}(t)|-1| \leqq \varepsilon \text {. }
$$

By Toponogov's comparison theorem (hereafter denoted by T. C. T.) ([5]), there exists $\alpha>0$ such that (5.1.1)

$$
\left|g\left(\dot{\sigma}(0), \dot{\bar{\gamma}}_{3}(0)\right)-1\right| \leqq \varepsilon / 10
$$

where $\sigma$ is a $\nabla$-geodesic from $\varphi(x)$ to $\tau(-\alpha)$. Let $\xi_{t}$ be a tangent vector at $\tau(t)$ of $\nabla$-geodesic from $\tau(-\alpha)$ to $\tau(t)$ and let $f(t)=g\left(\xi_{t}, \dot{\tau}(t)\right)$. Then, from the 
fact $|\tilde{\nabla} T| \leqq \delta$ and Proposition 4.1, we see

$$
\begin{aligned}
\left|\nabla_{\dot{\tau}(t)} \dot{\tau}(t)\right|= & \left|\nabla_{d \varphi\left(\dot{\gamma}_{3}(t)\right)} d \varphi\left(\dot{\gamma}_{3}(t)\right)\right| \\
\leqq & \mid \tilde{\nabla}_{d \varphi\left(\dot{\gamma}_{3}(t)\right)} d \varphi\left(\dot{\gamma}_{3}(t)\right)+T_{d \varphi\left(\dot{\gamma}_{3}(t)\right)} d \varphi\left(\dot{\gamma}_{3}(t) ;\right. \\
\leqq & \left|d \varphi\left(\tilde{\nabla}_{\dot{\gamma}_{3}(t)} \dot{\gamma}_{3}(t)\right)+d \varphi\left(T_{\dot{\gamma}_{3}(t)} \dot{\gamma}_{3}(t)\right)\right| \\
& +\left|d \varphi\left(\tilde{\nabla}_{\dot{\gamma}_{3}(t)} \dot{\gamma}_{3}(t)\right)-\tilde{\nabla}_{d \varphi\left(\dot{\gamma}_{3}(t)\right)} d \varphi\left(\dot{\gamma}_{3}(t)\right)\right| \\
& +\left|d \varphi\left(T_{\dot{\gamma}_{3}(t)} \dot{\gamma}_{3}(t)\right)-T_{d \varphi\left(\dot{\gamma}_{3}(t)\right)} d \varphi\left(\dot{\gamma}_{3}(t)\right)\right| \\
= & \left|d \varphi\left(\tilde{\nabla}_{\dot{\gamma}_{3}(t)} \dot{\gamma}_{3}(t)\right)-\tilde{\nabla}_{d \varphi\left(\dot{\gamma}_{3}(t)\right)} d \varphi\left(\dot{\gamma}_{3}(t)\right)\right| \\
& +\left|d \varphi\left(T_{\dot{\gamma}_{3}(t)} \dot{\gamma}_{3}(t)\right)-T_{d \varphi\left(\dot{\gamma}_{3}(t)\right)} d \varphi\left(\dot{\gamma}_{3}(t)\right)\right| \\
\leqq & C R \delta .
\end{aligned}
$$

On the other hand

$$
\left|g\left(\nabla_{\dot{\tau}(t)} \xi_{t}, \dot{\tau}(t)\right)\right|=\left|\operatorname{Hess} d_{\tau(-\alpha)}(\dot{\tau}(t), \dot{\tau}(t))\right|=\left|\operatorname{Hess} d_{\tau(-\alpha)}\left(\dot{\tau}(t)^{\perp}, \dot{\tau}(t)^{\perp}\right)\right|,
$$

where Hess $d_{\tau(-\alpha)}$ is the hessian of the distance function from $\tau(-\alpha)$ and $\dot{\tau}(t)^{\perp}$ is the component normal to $\xi_{t}$ of $\dot{\tau}(t)$. Hence, taking $\delta \leqq \varepsilon$ in Proposition 4.1, we see, by the hessian estimate [9] or [16] 1.4.4, that

$$
\begin{aligned}
& \left|g\left(\nabla_{\dot{\tau}(t)} \xi_{t}, \dot{\tau}(t)\right)\right| \\
\leqq & \left|\frac{1}{d(\tau(-\alpha), \tau(t))}+\frac{\Lambda}{2} d(\tau(-\alpha), \tau(t))\right||\dot{\tau}(t)|^{2}\left(1-g\left(\xi_{t}, \dot{\tau}(t) /|\dot{\tau}(t)|\right)\right) .
\end{aligned}
$$

Note that if $\hat{l}>0$ is sufficiently small, then

$$
d(\tau(-\alpha), \tau(t)) \geqq \alpha / 2 \quad \text { for } 0 \leqq t \leqq \hat{l}
$$

thus, by (5.1.1),

$$
\left|g\left(\nabla_{\dot{\tau}(t)} \xi_{t}, \dot{\tau}(t)\right)\right| \leqq\left(\frac{2}{\alpha}+\frac{\Lambda}{2}\right)(1+\delta)(1-(1-\delta) f(t)) .
$$

Next we show that $\hat{l}$ can be choosed as $\hat{l} \geqq l$. By (5.1.3) and (5.1.4), we find

$$
\left|\frac{d}{d t} f(t)\right| \leqq\left|g\left(\nabla_{\dot{\tau}(t)} \xi_{t}, \dot{\tau}(t)\right)\right|+\left|g\left(\xi_{t}, \nabla_{\dot{\tau}(t)} \dot{\tau}(t)\right)\right| \leqq C(1-(1-\delta) f(t))+C R \delta .
$$

This implies

$$
|1-f(t)| \leqq C^{\prime} R \delta \quad \text { for } 0 \leqq t \leqq \hat{l} .
$$

Assume that $\hat{l} \leqq l$. Then, $\left.(d / d t)\right|_{t=\hat{l}} d(\tau(-\alpha), \tau(t))=f(\hat{l})=0$. Thus, if we take $\delta \leqq \varepsilon / 10 C^{\prime} R$ in (5.1.5), then we show that $\hat{l}>l$ and

$$
|1-f(t)| \leqq \varepsilon / 10 \quad \text { for } 0 \leqq t \leqq l .
$$

Since $f(l)=g\left(\xi_{l}, \dot{\tau}(l)\right)=g\left(\dot{\sigma}(0), d \varphi\left(\dot{\gamma}_{3}(0)\right)\right)$, by Proposition 4.1, 


$$
\begin{aligned}
\left|1-g\left(\dot{\bar{\gamma}}_{3}(0), d \varphi\left(\dot{\gamma}_{3}(0)\right)\right)\right| \leqq & \left|1-g\left(\dot{\sigma}(0), d \varphi\left(\dot{\gamma}_{3}(0)\right)\right)\right| \\
& +\left|g\left(\dot{\sigma}(0), d \varphi\left(\dot{\gamma}_{3}(0)\right)\right)-g\left(\dot{\bar{\gamma}}_{3}(0), d \varphi\left(\dot{\gamma}_{3}(0)\right)\right)\right| \\
\leqq & \varepsilon / 10+\varepsilon / 10<\varepsilon / 2 .
\end{aligned}
$$

By Proposition 4.1, there exists $\delta>0$, if $M$ satisfies $(*)$ or $(* *)$ for $\delta$, then

$$
|| d \varphi\left(\dot{\gamma}_{3}(0)\right)|-| \dot{\tilde{\gamma}}_{3}(0)||<\varepsilon / 2 .
$$

Combining (5.1.7) and (5.1.8), we get the conclusion.

q. e. d.

Proposition 5.2. For any $\varepsilon>0$ and $R>0$, there exists $\delta=\delta(\varepsilon, R)>0$ such that if $M$ satisfies (*) or (**) for $\delta$, then

$$
d(\varphi(y), \phi(y)) \leqq \varepsilon \quad \text { for } y \in B_{r / 2}(p) .
$$

Proof. By Proposition 4.1, there exists $\delta>0$ such that if $M$ satisfies (*) or $(* *)$ for $\delta$, then

$$
\left|l_{3}-\tilde{l}_{3}\right| \leqq \varepsilon, \quad|| \dot{\gamma}_{3}(0)|-1| \leqq \varepsilon .
$$

We apply T.C.T. to the triangle with summits $\varphi(x)=\bar{\gamma}_{3}(0)=\tilde{\gamma}_{3}(0), \varphi(y)=\bar{\gamma}_{3}\left(\bar{l}_{3}\right)$ and $\tilde{\gamma}_{3}\left(l_{3}\right)$. Then, using also (5.1.7), we have

$$
d\left(\varphi(y), \tilde{\gamma}_{3}\left(l_{3}\right)\right)=d\left(\bar{\gamma}_{3}\left(\bar{l}_{3}\right), \tilde{\gamma}_{3}\left(l_{3}\right)\right) \leqq C \varepsilon .
$$

Let $\hat{\gamma}_{3}(t)$ be a $\nabla$-geodesic with $\hat{\gamma}_{3}(0)=\varphi(x)=\phi(x)$ and $\hat{\gamma}_{3}\left(\hat{l}_{3}\right)=\phi(y)$. If we replace $\varphi, \bar{\gamma}_{3}, I$ to $\phi, \hat{\gamma}_{3}, \widetilde{P}_{q \varphi(x)}^{\varphi\left(\gamma_{2}\right)} \circ I \circ \widetilde{P}_{x p}^{\gamma_{1}-1}$ in the proof of Lemma 5.1, then the same argument yields the following. There exists $\delta>0$ such that if $M$ satisfies (*) or (**) for $\delta$, then

$$
\left|g\left(\dot{\widetilde{\gamma}}_{3}(0), \dot{\gamma}_{3}(0)\right)-1\right| \leqq \varepsilon
$$

Combining with Lemma 5.1, by T.C. T., we get the conclusion.

Proposition 5.3. For any $\varepsilon>0$ and $R>0$, there exists $\delta=\delta(\varepsilon, R)>0$ such that if $M$ satisfies (*) or (**) for $\delta$, then

$$
\left|\Varangle\left(d_{y} \varphi(X), \tilde{P}_{\psi(y) \varphi(y)} d_{y} \psi(X)\right)\right| \leqq \varepsilon
$$

for each unit vector $X$ in $T_{y} M$, where $\Varangle(a, b)$ is the angle between $a$ and $b$.

Proof. Let $\gamma_{4}$ be a $\nabla$-geodesic with $\gamma_{4}(0)=y, \dot{\gamma}_{4}(0)=X$. Take $l_{4}>0$ satisfying $z=\gamma_{4}\left(l_{4}\right) \in B_{r / 2}(p)$. Let $\gamma_{5}, \gamma_{6}$ be $\nabla$-geodesics with $\gamma_{5}(0)=p, \gamma_{6}(0)=x$ and $\gamma_{5}\left(l_{5}\right)$ $=\gamma_{6}\left(l_{6}\right)=z$. Put

$$
\begin{aligned}
& \phi_{1}=\exp _{\psi(y)}(\tilde{\nabla}) \circ \tilde{P}_{q \psi(y)}^{\phi\left(\gamma_{1}\right)} \circ \tilde{P}_{q \varphi}^{\varphi\left(\gamma_{2}\right)} \circ I \circ \tilde{P}_{x p} \circ \widetilde{P}_{y x} \circ \exp _{y}(\tilde{\nabla})^{-1}, \\
& \phi_{2}=\exp _{\varphi(y)}(\tilde{\nabla}) \circ \tilde{P}_{q \varphi(y)}^{\varphi\left(\gamma_{1}\right)} \circ I \circ \tilde{P}_{y p} \circ \exp _{y}(\tilde{\nabla})^{-1} .
\end{aligned}
$$

We apply an argument of Proposition 5.2 successively. Replace the objects in 
Proposition 5.2 to the three cases by

(1) $R \rightarrow R, \quad p \rightarrow p, \quad x \rightarrow y, \quad y \rightarrow z, \quad \varphi \rightarrow \varphi, \quad \phi \rightarrow \psi_{2}, \quad \gamma_{1} \rightarrow \gamma_{5}, \quad \gamma_{2} \cup \gamma_{3} \rightarrow \gamma_{1} \cup \gamma_{4}$.

(2) $R \rightarrow R, \quad p \rightarrow p, \quad x \rightarrow x, \quad y \rightarrow z, \quad \varphi \rightarrow \varphi, \quad \phi \rightarrow \phi, \quad \gamma_{1} \rightarrow \gamma_{5}, \quad \gamma_{2} \cup \gamma_{3} \rightarrow \gamma_{2} \cup \gamma_{6}$.

(3) $R \rightarrow 2 R, \quad p \rightarrow x, \quad x \rightarrow y, \quad y \rightarrow z, \quad \varphi \rightarrow \phi, \quad \phi \rightarrow \psi_{1}, \quad \gamma_{1} \rightarrow \gamma_{6}, \quad \gamma_{2} \cup \gamma_{3} \rightarrow \gamma_{3} \cup \gamma_{4}$, $\sigma \rightarrow \gamma_{2}{ }^{-1} \cup \sigma \cup \varphi\left(\gamma_{2}\right) . \quad\left(\sigma\right.$ is in $\left.I=\tilde{P}_{p q}^{\sigma}.\right)$

Then we have

(1) $\quad d\left(\varphi(z), \psi_{2}(z)\right) \leqq \varepsilon / 10$

(2) $\quad d(\varphi(z), \psi(z)) \leqq \varepsilon / 10$

(3) $\quad d\left(\psi(z), \psi_{1}(z)\right) \leqq \varepsilon / 10$

and thus,

$$
d\left(\phi_{1}(z), \phi_{2}(z)\right) \leqq \varepsilon / 2 .
$$

Let $X_{1}=\tilde{P}_{\psi(y) \varphi(y)} d_{y} \psi(X)$ and $Y_{1}=P_{\psi(y) \varphi(y)} d_{y} \psi(X)$. Then by Lemma 3.4 and (5.3.1),

$$
\left|\Varangle\left(X_{1}, Y_{1}\right)\right| \leqq \Lambda \varepsilon \text {. }
$$

Let $\lambda, \mu, \nu$ be $\nabla$-geodesics with $\lambda(0)=\varphi(y), \dot{\lambda}(0)=X_{2}, \mu(0)=\phi_{1}(y)=\psi(y), \dot{\mu}(0)=$ $d_{y} \psi_{1}(y), \nu(0)=\phi_{2}(y)=\varphi(y)$ and $\dot{\nu}(0)=d_{y} \psi_{2}(X)$ and let $\bar{\mu}$ and $\bar{\nu}$ be $\nabla$-geodesics with $\bar{\mu}(0)=\phi_{1}(y), \bar{\mu}\left(l_{\mu}\right)=\phi_{1}(z), \bar{\nu}(0)=\phi_{2}(y)$ and $\bar{\nu}\left(l_{\nu}\right)=\phi_{2}(z)$. We apply an argument of Lemma 5.1, replacing $\gamma_{3}, \bar{\gamma}_{3}$ to $\mu, \bar{\mu}$ and $\nu, \bar{\nu}$, we see that there exists $\delta=$ $\delta(\varepsilon, R)>0$ such that if $M$ satisfies (*) or (**) for $\delta$, then

$$
|\Varangle(\dot{\mu}(0), \dot{\bar{\mu}}(0))| \leqq \varepsilon, \quad|\Varangle(\dot{\nu}(0), \dot{\bar{\nu}}(0))| \leqq \varepsilon .
$$

By T.C.T., we get

$$
d\left(\mu\left(l_{\mu}\right), \phi_{1}(z)\right) \leqq C \varepsilon, \quad d\left(\nu\left(l_{\nu}\right), \phi_{2}(z)\right) \leqq C \varepsilon .
$$

By [5], 1.31 Corollary, we get

$$
d\left(\lambda\left(l_{\nu}\right), \mu\left(l_{\nu}\right)\right) \leqq C \varepsilon .
$$

Thus, by (5.3.1), (5.3.3) and (5.3.4), we get

$$
\begin{aligned}
d\left(\lambda\left(l_{\mu}\right), \nu\left(l_{\nu}\right)\right) \leqq & d\left(\lambda\left(l_{\mu}\right), \mu\left(l_{\mu}\right)\right)+d\left(\mu\left(l_{\mu}\right), \phi_{1}(z)\right) \\
& +d\left(\phi_{1}(z), \phi_{2}(z)\right)+d\left(\phi_{2}(z), \nu\left(l_{\nu}\right)\right) \\
\leqq & C \varepsilon .
\end{aligned}
$$

Using Proposition 4.1 and T.C.T., we have

$$
\left|\Varangle\left(\dot{\lambda}(0), d_{y} \psi_{2}(X)\right)\right| \leqq \varepsilon .
$$


Note that $d_{y} \psi_{2}(X)=\tilde{P}_{q \varphi(y)}^{\varphi\left(\gamma_{1}\right)} \circ I \circ \tilde{P}_{y p}(X)$ for $X \in T_{y} M$, combining with Lemma 3. 4 and Proposition 4.1, we get

$$
\begin{aligned}
\left|\Varangle\left(d_{y} \varphi(X), \tilde{P}_{\psi(y) \varphi(y)} d_{y} \psi(X)\right)\right| & \leqq\left|\Varangle\left(d_{y} \psi_{2}(X), X_{1}\right)\right|+\left|\Varangle\left(d_{y} \varphi(X), d_{y} \psi_{2}(X)\right)\right| \\
& \leqq\left|\Varangle\left(d_{y} \psi_{2}(X), X_{1}\right)\right|+(1+\varepsilon) \varepsilon \\
& \leqq\left|\Varangle\left(d_{y} \psi_{2}(X), \dot{\lambda}(0)\right)\right|+2 \varepsilon+\left|\Varangle\left(X_{2}, X_{1}\right)\right| \leqq C \varepsilon .
\end{aligned}
$$

Hence we get the conclusion.

LEMMA 5.4. For any $\varepsilon>0$ and $R>0$, there exists $\delta=\delta(\varepsilon, R)>0$ such that if $M$ satisfies (*) or $(* *)$ for $\delta$, then the following holds. Take $q_{1}, q_{2} \in B_{r / 2}(p)$ satisfying the conditions (1), (2).

(1) There exist continuous curves $\sigma_{1}, \sigma_{2}$ with $l\left(\sigma_{i}\right) \leqq R,(i=1,2)$

$$
\begin{gathered}
\left|\Varangle\left(\tilde{P}_{p q_{1}}^{\sigma_{1}} X, \tilde{P}_{q_{2} q_{1}} \circ \tilde{P}_{p_{q_{2}}}^{\sigma_{2}} X\right)\right| \leqq \delta . \\
d\left(q_{1}, q_{2}\right) \leqq \delta .
\end{gathered}
$$

Put

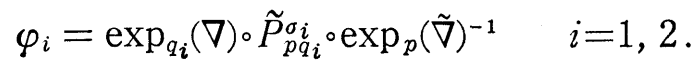

Then, $\varphi_{i}$ has the properties that

$$
\begin{aligned}
& || d_{x} \varphi_{i}(X)|-1| \leqq \varepsilon \quad i=1,2, \\
& d\left(\varphi_{1}(x), \varphi_{2}(x)\right) \leqq \varepsilon, \\
& \left|\Varangle\left(d_{x} \varphi_{1}(X), \widetilde{P}_{\varphi_{2}(x) \varphi_{1}(x)} d_{x} \varphi_{2}(X)\right)\right| \leqq \varepsilon,
\end{aligned}
$$

for unit vector $X \in T_{x} M$ and $x \in B_{r}(p)$.

PROOF. The conclusion (a) can be proved by the same argument to Proposition 4.1. Put $\varphi_{3}=\exp _{q_{1}}(\tilde{\nabla}) \circ \widetilde{P}_{q_{2} q_{1}}{ }^{\circ} \exp _{q_{2}}(\tilde{\nabla})^{-1}$ and $\hat{\varphi}_{1}=\varphi_{3} \circ \varphi_{2}$. By the assumption (1), we see that

$$
d\left(\varphi_{1}(x), \hat{\varphi}_{1}(x)\right) \leqq C \delta .
$$

To prove (b), we estimate $d\left(y, \varphi_{3}(y)\right)$. Let $\sigma_{1}, \sigma_{2}$ be $\nabla$-geodesics with $\sigma_{1}(0)=q_{2}$, $\sigma_{1}(l)=y, \sigma_{2}(0)=q_{1}$ and $\sigma_{2}(0)=P_{q_{2} q_{1}} \dot{\sigma}_{1}(0)$. By Lemma 3.4, Proposition 4.1 and [5], 1.31 Corollary, there exists $\delta=\delta(\varepsilon, R)>0$ such that if $M$ satisfies (*) or (**) for $\delta$, then,

$$
\begin{aligned}
& \left|\Varangle\left(P_{q_{2} q_{1}} \sigma_{1}(0), P_{q_{2} q_{1}} \sigma_{1}(0)\right)\right| \leqq \varepsilon, \\
& d\left(\varphi_{3}(y), \sigma_{2}(l)\right) \leqq \varepsilon, \quad d\left(y, \sigma_{2}(l)\right)=d\left(\sigma_{1}(l), \sigma_{2}(l)\right) \leqq \varepsilon .
\end{aligned}
$$

Thus, we have

$$
d\left(y, \varphi_{3}(y)\right) \leqq d\left(y, \sigma_{2}(l)\right)+d\left(\sigma_{2}(l), \varphi_{3}(y)\right) \leqq \varepsilon .
$$

Combining with (5.4.1) we get the conclusion (b). To prove (c), we put 


$$
\begin{aligned}
& \varphi_{4}=\exp _{\varphi_{1}(x)}(\tilde{\nabla}) \circ \tilde{P}_{q_{1} \varphi_{1}(x)}^{\varphi_{1}(\lambda)} \circ \tilde{P}_{p q_{1}}^{\sigma_{1}} \circ \tilde{P}_{x p}^{\lambda-1} \circ \exp _{x}(\tilde{\nabla})^{-1}, \\
& \varphi_{5}=\exp _{\varphi_{2}(x)}(\tilde{\nabla}) \circ \tilde{P}_{q_{2} \varphi_{2}(x)}^{\varphi_{2}(\lambda)} \circ \tilde{P}_{p_{2}}^{\sigma_{2}} \circ \tilde{P}_{x p}^{\lambda-1} \circ \exp _{x}(\tilde{\nabla})^{-1}
\end{aligned}
$$

where $\lambda$ is a $\nabla$-geodesic from $p$ to $x$. Replace $\varphi$ and $\phi$ in Proposition 4.2 to $\varphi_{1}$ and $\varphi_{4}$, or $\varphi_{2}$ and $\varphi_{5}$. We see that there exists $\delta=\delta(\varepsilon, R)>0$ such that if $M$ satisfies $(*)$ or $(* *)$ for $\delta$, then

$$
d\left(\varphi_{1}(z), \varphi_{4}(z)\right) \leqq \varepsilon, \quad d\left(\varphi_{2}(z), \varphi_{5}(z)\right) \leqq \varepsilon .
$$

By the conclusion (a), we have

$$
\begin{aligned}
d\left(\varphi_{4}(z), \varphi_{5}(z)\right) & \leqq d\left(\varphi_{4}(z), \varphi_{1}(z)\right)+d\left(\varphi_{1}(z), \varphi_{2}(z)\right)+d\left(\varphi_{2}(z), \varphi_{5}(z)\right) \\
& \leqq 2 \varepsilon+C \delta .
\end{aligned}
$$

Let $\mu_{1}, \mu_{2}$ be $\nabla$-geodesics from $\varphi_{1}(x)=\varphi_{4}(x)$ to $\varphi_{4}(z)$, from $\varphi_{2}(x)$ to $\varphi_{5}(x)$. By [5], 1.31 Corollary, Lemma 3, 4 and Proposition 4.2, combining with (b) there exists $\delta=\delta(\varepsilon, R)>0$ such that if $M$ satisfies $(*)$ or $(* *)$ for $\delta$, then

$$
\begin{aligned}
& \left|P_{\varphi_{2}(x) \varphi_{1}(x)} \dot{\mu}_{1}(0)-\dot{\mu}_{2}(0)\right| \leqq \varepsilon, \\
& \left|\Varangle\left(P_{\varphi_{2}(x) \varphi_{1}(x)} \dot{\mu}_{1}(0), \widetilde{P}_{\varphi_{2}(x) \varphi_{1}(x)} \dot{\mu}_{1}(0)\right)\right| \leqq \varepsilon, \\
& \left|\mu_{i}(0)-d_{x} \varphi_{i}(X)\right| \leqq \varepsilon, \quad i=1,2 .
\end{aligned}
$$

Hence the conclusion.

A broken $\nabla$-geodesic is a continuous curve $\gamma:[0, l] \rightarrow M$ such that there exist $0<t_{0}<\cdots<t_{n}<t_{n+1}=l$ and $\left.\gamma\right|_{\left[t_{i}, t_{i+1}\right]}$ is a $\nabla$-geodesic. Put $\gamma(0)=p$ and ${ }_{i} \gamma=$

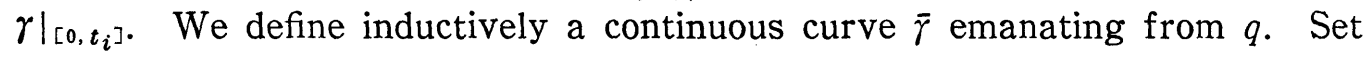

$$
{ }_{1} \bar{\gamma}(t)=\exp _{q}(\tilde{\nabla}) \circ I \circ \exp _{p}(\tilde{\nabla})^{-1}(\gamma(t)) .
$$

Assume that $i \bar{\gamma}$ has already been defined. Set

and

$$
\Phi\left[{ }_{i} \gamma\right]=\exp _{i \bar{\gamma}\left(t_{i}\right)}(\tilde{\nabla}) \circ \tilde{P}_{q \bar{\gamma}\left(t_{i}\right)}^{i r} \circ I^{\circ} \tilde{P}_{\gamma\left(t_{i}\right) p^{\circ}}^{i^{-1}} \exp _{\gamma\left(t_{i}\right)}(\tilde{\nabla})^{-1}
$$

$$
{ }_{i+1} \bar{\gamma}(t)= \begin{cases}\bar{i}(t) & 0 \leqq t \leqq t_{i} \\ \Phi\left[{ }_{i} \gamma\right](\gamma(t)) & t_{i} \leqq t \leqq t_{i+1}\end{cases}
$$

Proposition 5.5. For any $\varepsilon>0$ and $R>0$, there exists $\delta=\delta(\varepsilon, R)>0$ such that if $M$ satisfies (*) or (**) for $\delta$, then the following holds. If broken $\nabla$ geodesics $\gamma_{1}, \gamma_{2}$ parametrized by arclength, satisfy

(1) $\gamma_{1}(0)=\gamma_{2}(0)=p, \quad \gamma_{j}\left(l_{j}\right)=z \quad(j=1,2)$,

(2) for broken points $\gamma_{j}\left(t_{i, j}\right) \quad(i=1,2, \cdots, n)$

$$
\gamma_{1}\left(t_{i+k, 1}\right), \gamma_{2}\left(t_{i+k, 2}\right) \in B_{r / 2}\left(\gamma_{j}\left(t_{i, j}\right)\right) \quad(k= \pm 1),
$$

(3) $l\left(\gamma_{j}\right) \leqq R$,

then, 
(i ) $d\left(\Phi\left[\gamma_{1}\right](z), \Phi\left[\gamma_{2}\right](z)\right) \leqq \varepsilon$

(ii) $\left|d_{z} \Phi\left[\gamma_{1}\right](X)-\widetilde{P}_{21} d_{z} \Phi\left[\gamma_{2}\right](X)\right| \leqq \varepsilon$

(iii) ||$d \Phi\left[\gamma_{j}\right](x)|-1| \leqq \varepsilon$,

for unit vector $X \in T_{z} M$, where $\tilde{P}_{j_{k}}$ is $\tilde{\nabla}$-parallel translation from $\Phi\left[\gamma_{2}\right](z)$ to $\Phi\left[\gamma_{1}\right](z)$ along minimal geodesic.

Proof. Step 1. First we show the result for $n=1$. Assume that $\gamma_{1}\left(t_{0,1}\right)$ $\neq \gamma_{2}\left(t_{0,2}\right), \gamma_{1}\left(t_{1,1}\right)=\gamma_{2}\left(t_{1,2}\right)=z$. Then by Propositions 5.2 and 5.3 , there exists $\delta=$ $\delta(\varepsilon, R)>0$ such that if $M$ satisfies $(*)$ or $(* *)$ for $\delta$, then

$$
d\left(\Phi\left[\gamma_{j}\right](z), \Phi\left[\gamma_{3}\right](z)\right) \leqq \varepsilon / 10, \quad\left|d_{z} \Phi\left[\gamma_{j}\right](X)-\widetilde{P}_{3 j} d_{z} \Phi\left[\gamma_{3}\right](X)\right| \leqq \varepsilon / 10 .
$$

Thus

(5.5.1) $d\left(\Phi\left[\gamma_{1}\right](z), \Phi\left[\gamma_{2}\right](z)\right) \leqq d\left(\Phi\left[\gamma_{1}\right](z), \Phi\left[\gamma_{3}\right](z)\right)+d\left(\Phi\left[\gamma_{3}\right](z), \Phi\left[\gamma_{2}\right](z)\right) \leqq \varepsilon / 5$.

On the other hand, by Proposition 4.1 and (5.5.1), there exists $\delta=\delta(\varepsilon, R)>0$, such that if $M$ satisfies $(*)$ or $(* *)$ for $\delta$, then

$$
\left|\widetilde{P}_{12} \circ \tilde{P}_{32} X-\tilde{P}_{31} X\right| \leqq \varepsilon / 10, \quad|| d_{z} \Phi\left[\gamma_{j}\right](X)|-1| \leqq \varepsilon / 10, \quad|| \tilde{P}_{j_{k}} X|-1| \leqq \varepsilon / 10 .
$$

Hence,

$$
\begin{aligned}
& \left|d_{z} \Phi\left[\gamma_{1}\right](X)-\widetilde{P}_{21} d_{z} \Phi\left[\gamma_{2}\right](X)\right| \\
\leqq & \left|d_{z} \Phi\left[\gamma_{1}\right](X)-\widetilde{P}_{31} d_{z} \Phi\left[\gamma_{3}\right](X)\right|+\left|\widetilde{P}_{21} \circ\left(\tilde{P}_{12} \circ \widetilde{P}_{31} d_{z} \Phi\left[\gamma_{3}\right](X)-\widetilde{P}_{32} d_{z} \Phi\left[\gamma_{3}\right](X)\right)\right| \\
& \left.+\mid \widetilde{P}_{21} \circ \widetilde{P}_{32} d_{z} \Phi\left[\gamma_{3}\right](X)-d_{z} \Phi\left[\gamma_{2}\right](X)\right) \mid \\
\leqq & \varepsilon / 10+2(1+(\varepsilon / 10)) \varepsilon / 10 \leqq \varepsilon .
\end{aligned}
$$

Step 2. Assume that the conclusion holds for $n-1$. Let $\tau:\left[t_{n-1}^{\prime}, t_{n}^{\prime}\right] \rightarrow M$ be a $\nabla$-geodesic with $\tau\left(t_{n-1}^{\prime}\right)=\gamma_{1}\left(t_{n-1,1}\right), \tau\left(t_{n}^{\prime}\right)=\gamma_{2}\left(t_{n, 2}\right)$. Put $\Phi\left[{ }_{n-1} \gamma_{1}\right]\left(\tau\left(t_{n}^{\prime}\right)\right)=z_{1}$ and $\Phi\left[{ }_{n-1} \gamma_{2}\right]\left(\gamma_{2}\left(t_{n}\right)\right)=z_{2}$. Then by the induction hypothesis, there exists $\delta=\delta(\varepsilon, R)$ $>0$ such that if $M$ satisfies (*) or (**) for $\delta$, then

$$
\begin{gathered}
d\left(z_{1}, z_{2}\right) \leqq \varepsilon / 10, \\
\left|d_{z_{1}} \Phi\left[{ }_{n-1} \gamma_{1}\right](X)-\widetilde{P}_{z_{2} z_{1}} d_{z_{2}} \Phi\left[{ }_{n-1} \gamma_{2}\right](X)\right| \leqq \varepsilon / 10, \\
\left|d_{z_{1}} \Phi\left[{ }_{n-1} \gamma_{1}\right](X)\right|-1 \mid \leqq \varepsilon / 10 .
\end{gathered}
$$

We put $\sigma_{1}=\left.\gamma_{1}\right|_{\left[t_{n-1,1}, l_{1}\right]}, \theta_{2}=\left.\gamma_{2}\right|_{\left[t_{n, 2}, l_{2}\right]}, \Phi\left[{ }_{n-1} \gamma_{1} \cup \tau \cup \theta_{2}\right]\left(\theta_{2}\left(l_{2}\right)\right)=\Phi\left[{ }_{n-1} \gamma_{1} \cup \tau \cup \theta_{2}\right](z)$ $=z_{3}$ and $\Phi\left[{ }_{n-1} \gamma_{1} \cup \sigma_{1}\right]\left(\gamma_{1}\left(l_{1}\right)\right)=\Phi\left[\gamma_{1}\right](z)=z_{4}$. Apply Step 1 to $\tau \cup \theta_{2}$ and $\sigma_{1}$. Then there exists $\delta=\delta(\varepsilon, R)>0$ such that if $M$ satisfies (*) or (**) for $\delta$,

$$
\begin{gathered}
d\left(z_{3}, z_{4}\right) \leqq \varepsilon / 10, \\
\left|d_{z} \Phi\left[{ }_{n-1} \gamma_{1} \cup \tau \cup \theta_{2}\right](X)-\widetilde{P}_{z_{4^{2}}} d_{z} \Phi\left[\gamma_{1}\right](X)\right| \leqq \varepsilon / 10, \\
|| d_{z} \Phi\left[{ }_{n-1} \gamma_{1} \cup \sigma_{1}\right](X)|-1| \leqq \varepsilon / 10, \\
|| d_{z} \Phi\left[{ }_{n-1} \gamma_{1} \cup \tau \cup \theta_{1}\right](X)|-1| \leqq \varepsilon / 10 .
\end{gathered}
$$


We apply Proposition 5.4 for $q_{1}=z_{1}, q_{2}=z_{2}$. Put $\Phi\left[\gamma_{2}\right]\left(\gamma_{2}\left(l_{2}\right)\right)=\Phi\left[\gamma_{2}\right](z)=z_{5}$. From (5.5.2), there exists $\delta=\delta(\varepsilon, R)>0$ such that if $M$ satisfies (*) or (**) for $\delta$, then

$$
\begin{gathered}
d\left(z_{3}, z_{5}\right) \leqq \varepsilon / 10, \\
\left|d_{z} \Phi\left[\gamma_{2}\right](X)-P_{z_{5} z_{3}} d \Phi\left[{ }_{n-1} \gamma_{1} \cup \tau \cup \theta_{2}\right](X)\right| \leqq \varepsilon / 10 .
\end{gathered}
$$

Therefore, by Proposition 4.1, there exists $\delta=\delta(\varepsilon, R)>0$ such that if $M$ satisfies $(*)$ or $(* *)$ for $\delta$, then

$$
\begin{aligned}
& d\left(\Phi\left[\gamma_{1}\right](z), \Phi\left[\gamma_{2}\right](z)\right) \leqq d\left(z_{4}, z_{3}\right)+d\left(z_{3}, z_{5}\right) \leqq \varepsilon / 5, \\
& \left|\widetilde{P}_{z_{3} z_{4}}{ }^{\circ} \widetilde{P}_{z_{5} z_{3}} d_{z} \Phi\left[\gamma_{2}\right](X)-\widetilde{P}_{z_{5} z_{4}} d_{z} \Phi\left[\gamma_{2}\right](X)\right| \leqq \varepsilon / 10 .
\end{aligned}
$$

Hence we get, from (5.5.3), (5.5.4) and (5.5.5),

$$
\begin{aligned}
&\left|d_{z} \Phi\left[\gamma_{1}\right](X)-\tilde{P}_{21} d_{z} \Phi\left[\gamma_{2}\right](X)\right| \\
&=\left|d_{z} \Phi\left[\gamma_{1}\right](X)-\tilde{P}_{z_{5} z_{4}} d_{z} \Phi\left[\gamma_{2}\right](X)\right| \\
& \leqq\left|d_{z} \Phi\left[\gamma_{1}\right](X)-\tilde{P}_{z_{3} z_{4}} d_{z} \Phi\left[n_{-1} \gamma_{1} \cup \tau \cup \theta_{2}\right](X)\right| \\
&+\left|\tilde{P}_{z_{3} z_{4}} d_{z} \Phi\left[{ }_{n-1} \gamma_{1} \cup \tau \cup \theta_{2}\right](X)-\tilde{P}_{z_{3} z_{4}} \tilde{P}_{z_{5} z_{4}} d_{z} \Phi\left[\gamma_{2}\right](X)\right| \\
&+\left|\tilde{P}_{z_{3} z_{4}} \tilde{P}_{z_{5} z_{3}} d_{z} \Phi\left[\gamma_{2}\right](X)-\tilde{P}_{z_{5} z_{4}} d_{z} \Phi\left[\gamma_{2}\right](X)\right| \\
& \leqq \varepsilon / 10+\varepsilon / 5+\varepsilon / 10 \leqq \varepsilon .
\end{aligned}
$$

Proposition 4.1 implies (iii).

q. e. d.

\section{§6. Proof of Theorem 2.}

As we show in Section 2, it sufficies to prove $(\mathrm{F})$. Fix $p, q \in \tilde{M}$, we prove that there exists a distance-preserving map $\Phi$ of $\tilde{M}$ with $\Phi(p)=q$. Let $f_{i}$ be a diffeomorphism from $\tilde{M}_{i}$ to $\tilde{M}$. Choose $r / 10$-dense, $r / 100$-discrete subset $N=\left\{p_{k}\right\}$ in $\tilde{M}$. Fix $x \in \tilde{M}$ and choose a sequence $J=\left\{p_{j}\right\}_{j=1}^{m} \subset N$ such that

$$
d\left(p, p_{1}\right) \leqq r / 10, \quad d\left(p_{j}, p_{j+1}\right) \leqq r / 10, \quad d\left(p_{m}, x\right) \leqq r / 10 .
$$

Let $\gamma^{i}$ a broken geodesic in $\tilde{M}_{i}$ with broken points $\left\{p_{j}\right\}$. We define

$$
\Phi_{i}^{J}=f_{i} \circ \Phi\left[\gamma^{i}\right] \circ f_{i}^{-1}
$$

Then, $\left\{\Phi_{i}^{J}\right\}$ are equicontinuous and uniformly bounded on $f_{i}^{-1}\left(B_{r / 2}\left(p_{j}\right)\right)$ by Theorem B. Hence Ascoli's theorem implies that there exists a limit $\Phi^{J}$. By Proposition 5.5, $\Phi^{J}$ is local distance preserving map on $B_{r / 3}\left(p_{m}\right)$. In the following, we show that $\Phi^{J}$ is independent of choosing,

(1) $\gamma^{i}$, 
(2)

$J$.

(1) can immediately follow. To prove (2), we take sequences $J_{1}=\left\{p_{j}\right\}$ and $J_{2}=$ $\left\{q_{k}\right\}$ satisfying the above condition (6.1). Since $\tilde{M}$ is simply connected, we can choose sequences $J[n], n=1,2, \cdots, l$ such that

$$
\begin{aligned}
& J[n]=\left\{p_{j, n}\right\}, \quad J[1]=J_{1}, \quad J[l]=J_{2}, \\
& p_{j+j^{\prime}, n+n^{\prime}} \in B_{r / 2}\left(p_{j, n}\right), \quad j^{\prime}, n^{\prime}= \pm 1 .
\end{aligned}
$$

From Proposition 5.5, we see,

$$
\lim _{i \rightarrow \infty} d\left(\Phi_{i}^{J[n]}(x), \Phi_{i}^{J[n+1]}\right)=0 .
$$

Thus, $\Phi^{J_{1}}=\Phi^{J_{2}}$. This implies (2). So we can define a map $\Phi=\Phi^{J}$ globally.

Finally we show that there exists $\Psi \in G_{0}$ with $\Psi(p)=q$. Let $\sigma(t)$ be a $\nabla$ geodesic with $\sigma(0)=p, \sigma(1)=q$. Then, we can define a map $\Phi_{t}$ by replacing $q$ in the definition of $\Phi$ to $q_{t}=\sigma(t)$. Note that $\Phi_{1}=\Phi$. If we put $\Psi=\Phi \circ \Phi_{0}{ }^{-1}$, then $\Psi \in G_{0}$.

q.e.d.

\section{§ 7. Proof of Theorem 1 .}

By the interpolation inequalties ([10], 12.1 Theorem and 12.5 Corollary), we see $|\nabla R| \leqq C_{m}\left|\nabla^{m} R\right|^{1 / m}$ for some constant $C_{m}$. Thus it suffices to prove in the case $m=1$. Substitute $T=0$ in Theorem 2, Then the limit manifold $M$ is $\left(C^{\infty}\right.$-)locally homogeneous space. To see that $M$ is locally symmetric, it sufficies to see that the geodesic symmetry of a neighborhood of a point of $M_{i}$ converges to the geodesic symmetry of a neighborhood of $M$ and that the limit is an isometry.

q. e.d.

\section{References}

[1] W. Ambrose and I. M. Singer, On homogeneous Riemannian manifolds, Duke Math. J., 25 (1958), 647-669.

[2] M. Berger, Sur les variétés riemannienes pincées juste audessous de 1/4, Ann. Inst. Fourier, 33 (1983), 135-150.

[3] J. Cheeger, Comparison and finiteness theorems for Riemannian manifolds, $\mathrm{Ph} . \mathrm{D}$. Thesis, Princeton Univ., 1967.

[4] — Finiteness theorems for Riemannian manifolds, Amer. J. Math., 92 (1970), 61-74.

[5] J. Cheeger and D. G. Ebin, Comparison Theorems in Riemannian Geometry, American Elsevier, Now York, 1975.

[6] K. Fukaya, Theory of convergence for Riemannian orbifolds, Japan J. Math., 12 (1986), 121-160.

[7] R.E. Greene and H. Wu, Lipshitz convergence of Riemannian manifolds, Pacific J. Math., 131 (1988), 119-141. 
[8] M. Gromov, Manifolds of negative curvature, J. Diff. Geom., 13 (1978), 223-230.

[9] - Structures métriques pour les variétés riemanniennes, rédigé par J. Lafontaine et P. Pansu, Cedic/Fernand Nathan, 1981.

[10) R. Hamilton, Three manifolds with positive Ricci curvature, J. Diff. Geom., 17 (1982), $255-306$.

[11] A. Katsuda, Gromov's convergence theorem and its application, Nagoya Math. J., 100 (1985), 11-48.

[12] S. Kobayashi and K. Nomizu, Foundations of Differential Geometry, John Wiley, New York, I, 1963, II, 1969.

[13] Min-Oo and E. Ruh, Comparison theorems for compact symmetric spaces, Ann. Sci. École Norm. Sup., 12 (1979), 335-353.

[14] — Vanishing theorems and almost symmetric spaces of noncompact type, Math. Ann., 257 (1981), 419-433.

[15] D. Montgomery and L. Zippin, Topological Transformation Groups, Interscience, 1955.

[16] T. Sakai, Comparison and finiteness theorems in Riemannian geometry, Adv. Stud. Pure Math., 3, Geometry of Geodesics, pp. 125-181.

[17] F. Tricerri and L. Vanhecke, Homogeneous Structures on Riemannian Manifolds, London Math. Soc., Lecture Note Ser., 83, Cambridge Univ. Press, 1983.

[18] S. Peters, Convergence of Riemannian manifolds, Compositio Math., 62 (1987), 3-16.

Atsushi Katsuda

Department of Mathematics

Okayama University

Okayama 700

Japan 